\title{
Flow Interference in Bluff Body Wakes
}

\author{
Rodrigo Surmas, Luís Orlando Emerich dos Santos, and Paulo Cesar Philippi \\ Mechanical Engineering Department, Federal University of Santa Catarina \\ Address: PB 476 88040-900 Florianópolis SC Brazil \\ \{philippi, surmas, emerich\}@1mpt.ufsc.br
}

\begin{abstract}
This paper presents a two-dimensional numerical simulation of fluid flow around a couple of identical circular cylinders aligned, respectively, along and orthogonal to the main-flow direction, at several distances. A latticeBoltzmann method (LB) is used. The forces resulting from fluid-solid interaction are calculated by considering the momentum exchanged between the fluid and the solid surfaces. Bluff body wake interference and its effect on drag and lift forces is studied in detail. The drag and the lift coefficients are obtained and compared with others existing results. A transition regime is obtained when the cylinders are aligned orthogonal to the main flow direction and separated by distances smaller than about 2D.
\end{abstract}

\section{Introduction}

The study of the flow pattern around bluff bodies is a subject of permanent interest and has, for a long time, attracted the attention of researchers, considering its increasing complexity with the Reynolds number, Re, and its great technological importance in engineering design.

For a single cylinder, fluid flow induces the appearance of vortex just behind any solid obstacle that is placed against it. When $\mathrm{Re}$ is greater than $\sim 45$, this flow pattern becomes mechanically unstable and vortices are shed off by the main flow, forming the von Karmann vortex street, [1].

In this way, after vortex street formation, the obstacle is subjected to a fluctuating drag that induces vibrations with frequencies that are related to the frequencies these vortices are formed and released to the wake.

A Fourier analysis of the lifting force shows that the vortex is formed with a single frequency when $\operatorname{Re}$ is near its mechanical stability limit $(\operatorname{Re} 45)$. When $\operatorname{Re}$ increases far from this limit, flow pattern changes to a transition pattern, [2,3], and its vortex formation frequency spectrum becomes broader.

Present paper deals with the vortex street interference process and its dynamic effects on the forces that two solid objects are subjected when placed at near locations, against the flow, [4-6].

Vortex street interference is a very interesting subject of growing scientific interest, since interference can lower the Reynolds limits to transition. In addition, flow interference between two circular cylinders is important in several applications. In 
petroleum engineering, interference can raise damage in bundles of risers in the offshore platforms of oil exploitation.

We restrict ourselves to two-dimensional incompressible flow around cylindrical shaped obstacles.

Flow is simulated using lattice Boltzmann equation, with a BGK, single relaxation time, collision model, [7-10].

The use of lattice-Boltzmann as a working-method in this class of problems has some important, well-known, advantages: i) programming is very easy and ii) the method is intrinsically parallel. Nevertheless, it appears that the most important advantage of the LB method with respect to classical CFD methods is related to the needless of velocity field numerical derivatives in calculating the drag force. In fact, due to its mesoscale nature, drag force can be directly calculated in LB methods by, simply, considering the momentum exchanged between the lattice-particles and the solid body.

\section{Lattice-Boltzmann Simulation}

As usually, let $\vec{c}_{i}$ indicate the lattice unitary-velocity along direction i of a D2Q9 lattice, i.e., a two dimensional 9-velocity lattice, which unitary cell is a square formed by the four, $\left|\vec{c}_{i}\right|=1$, principal directions, by the four, $\left|\vec{c}_{i}\right|=\sqrt{2}$, diagonals and by one $\left|\vec{c}_{i}\right|=0$. Let $N_{i}(\vec{X}, T)$ designate the particles distribution in the i-direction of site $\vec{X}$, at time T. Lattice-Boltzmann equation with BGK collision model is written as

$$
N_{i}\left(\vec{X}+\vec{c}_{i}, T+1\right)-N_{i}(\vec{X}, T)=\frac{N_{i}^{e q}(\vec{X}, T)-N_{i}(\vec{X}, T)}{\tau},
$$

where $\tau$ is a relaxation time, related to fluid kinematics viscosity and $N_{i}^{e q}(\vec{X}, T)$ is an equilibrium distribution that can be considered as a D2Q9 gaussian quadrature of the Maxwell-Boltzmann continuous distribution function, [8], and is given by

$$
N_{i}^{e q}=w_{i} \rho\left\{1+\frac{\left(c_{i} \cdot u\right)}{c_{s}^{2}}+\frac{\left(c_{i} \cdot u\right)^{2}}{2 c_{s}^{4}}-\frac{u^{2}}{2 c_{s}^{2}}\right\}
$$

where is $w_{i}=1 / 9$ for the principal directions, $\mathrm{i}=1,2,3,4 ; w_{i}=1 / 36$ for the diagonals, $\mathrm{i}=5,6,7,8$; and $w_{0}=4 / 9 . c_{s}^{2}=1 / 3$ is the square of LB sound velocity in a D2Q9 lattice.

At each simulation time-step, velocity field $\vec{u}(\vec{X}, T)$ and pressure $P(\vec{X}, T)$ are, respectively, calculated by using

$$
\rho \vec{u}=\sum_{i} N_{i} \vec{c}_{i}, \quad P=c_{s}^{2} \sum_{i} N_{i}=c_{s}^{2} \rho,
$$


Boundary conditions were taken as the following: $u_{x}=U, u_{y}=0$ at the left $(x=0)$ and the right $\left(x=L_{x}\right)$ and at the upper and lower boundaries of the simulation domain. $\mathrm{U}$ is the main-flow velocity.

Reynolds number is calculated by using

$$
R e=\frac{U D}{v},
$$

where $D$ is the cylinder diameter and $v=\frac{\left(\tau-\frac{1}{2}\right)}{3}$ is the LB kinematic viscosity in a D2Q9 lattice.

Flow adherence at solid boundaries is simulated by using the bounce-back condition, i.e., by requiring that $N_{i}\left(\vec{X}_{b}, T+1\right)=N_{-i}\left(\vec{X}_{b}, T\right)$ for all the b-sites at the fluid domain closest to the solid-boundary sites and for all the directions i, related to these sites, pointing outward the solid surface.

Considering that discrete circles have a discreteness-produced rough surface, the use of bounce-back conditions at this rough surface, requires the enlargement of the simulation domain until simulation results become insensible to discreteness effects.

This is an important question in CFD simulation, considering computational costs. Generalized coordinates are used in association with finite-volumes method, but mesh generation is computationally expensive and the method is highly subjected to stability problems.

Since He and Luo, [4], has demonstrated that LB equation is a special discrete form of the Boltzmann continuous equation, some effort has been undertaken in LB simulation, for the implementation of non-regular lattices and/or varying-size mesh, [11]. For varying size mesh, interpolation is required and interpolation has been shown to increase stability problems, [12].

The strong development of computers is, presently, pulling-back some classical CFD practitioners to Cartesian mesh, with larger resolution. An important contribution in this field was given by Lima e Silva et al. (2002), [4], with the immersed boundary method.

In this sense, present work is limited to the regular D2Q9 lattice.

The total force that the flow exerts on a solid body which boundary surface is $\Gamma$ is calculated by

$$
\vec{F}=\sum_{\Gamma} \sum_{i}\left\lfloor N_{-i}\left(\vec{X}_{\Gamma}, T^{+}\right)+N_{i}\left(\vec{X}_{\Gamma}, T^{-}\right)\right] \vec{c}_{i},
$$

i.e., by calculating the momentum variation of each site $\vec{X}_{\Gamma}$ in the fluid domain contiguous to the boundary surface, considering the particles $N_{i}\left(\vec{X}_{\Gamma}, T^{-}\right)$in the $\mathrm{i}=$ direction pointing to the surface, before propagation, that were bounced back to the direction -i, after propagation, $N_{-i}\left(\vec{X}_{\Gamma}, T^{+}\right)$.

The drag and the lift forces are, respectively, the $x$ and $y$ components of $\vec{F}$, when $x$ is the main-flow direction. 
When the flow pattern is symmetric with respect to the main-flow direction $x$, the lift-force is zero. This is what happens with immersed cylinders at very small Reynolds number. When $\mathrm{Re}$ is increased, vortex shedding behind the cylinder produces an oscillating lift with the same frequency of the vortices that are formed.

An average drag coefficient $\bar{C}_{D}$ was calculated as the time average of

$$
C_{D}(T)=\frac{F_{x}(T)}{\frac{1}{2} \rho U^{2} D} .
$$

Lift coefficient $C_{L}$ is calculated in the same way, but using the main-flow orthogonal component $F_{y} . C_{L}$ is also a function of time and, frequently, $\bar{C}_{L}=0$. When the cylinders are disposed in tandem, a root mean square was used for evaluate the lift, calculated as,

$$
C_{L}^{r m s}=\sqrt{\frac{\sum_{T}^{T+N}\left[c_{L}(T)-\bar{c}_{L}\right]^{2}}{N}} .
$$

\section{Results}

\subsection{Single Circular Cylinder}

Preliminary tests were performed for the single cylinder problem, with the purpose of validating the present LB simulation sheme, in what it concerns drag force (Eq. 5) and Strouhal number calculations. Diferent lattice sizes were tested from $334 \times 200$ to $3000 \times 1800$ lattice units, considering a cylinder with 40 lattice units along ist diameter. A 1000x600 lattice was considered to give enough accurate results. Comparisons with some others authors, [13-18], are presented in Fig. 1 showing a very good agreement for both the drag and the Strouhal number.

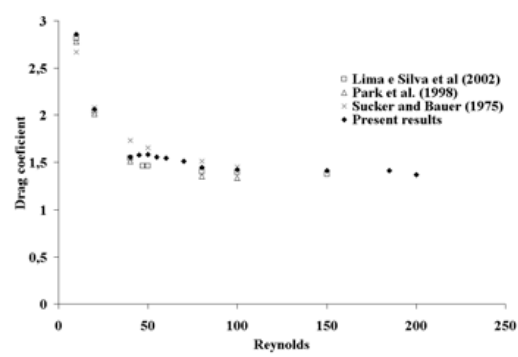

a) Drag Coeficient

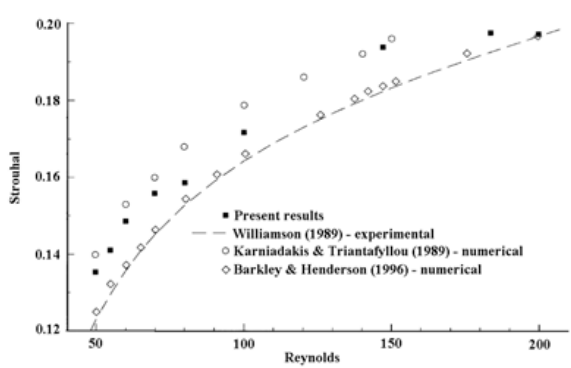

b) Strouhal number

Fig. 1. Results of one cylinder placed in the flow. 


\subsection{Two Identical Circular Cylinders Aligned along the Main Flow Direction}

Fig. 2 shows the simulation domain and the geometric parameters that were used in the LB simulation. Distance L between the centers of the two cylinders, referred as 1 and 2 was varied from $1.5 \mathrm{D}$ to $4 \mathrm{D}$.

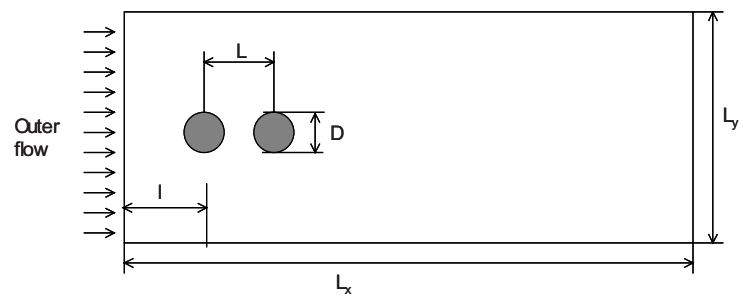

Fig. 2. Simulation domain for the fluid flow against two cylinders disposed in tandem. $\mathrm{L}_{\mathrm{x}}=$ 33D (1320 lattice sites), $\mathrm{L}_{\mathrm{y}}=15 \mathrm{D}$ (600 lattice sites), $\mathrm{L}=11 \mathrm{D}, \mathrm{D}=40$ lattice-units. Distance $\mathrm{L}$ between the centers of the two cylinders was varied from $1.5 \mathrm{D}$ to $4 \mathrm{D}$

Fig. 3 shows the vorticity patterns for several L. When $\mathrm{L}$ is smaller than $23.5 \mathrm{D}$, vortices that were formed in the intermediate region between the upstream and the downstream cylinders are not shed off until those vortices reach the downstream cylinder. A low pressure are formed between the two cylinders. Therefore, this low pressure region induces a negative drag on the downstream cylinder. This cylinder is attracted to the upstream body, [5,6].

This can be better seen in Fig. 4, which presents the simulation results for the drag, $\bar{C}_{D}$ and lift, $\bar{C}_{L}^{r m s}$, coefficients, for the two cylinders. Drag results were compared with Meneghini et al.'s results, [5], who have used a fractional step method. Agreement between the two methods can be considered as good, although Meneghini and co-authors did not report any data for $\mathrm{L}=2.5 \mathrm{D}$ or $3.5 \mathrm{D}$. Although results are very dissimilar for $\mathrm{L}=4.0 \mathrm{D}$, there is a good agreement between both works for the transition point, when the drag becomes positive for the downstream cylinder. For the upstream cylinder, drag forces appear to be overestimated in Lattice Boltzmann simulations that have been performed with $1320 \times 600$ lattice.

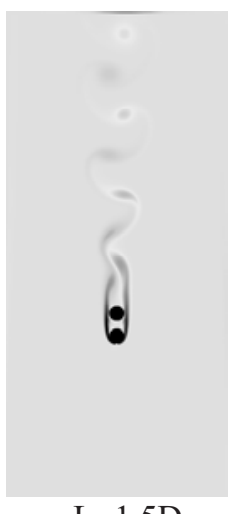

$\mathrm{L}=1.5 \mathrm{D}$

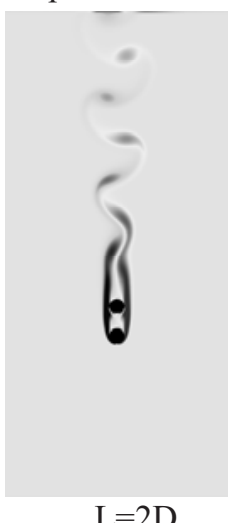

$\mathrm{L}=2 \mathrm{D}$
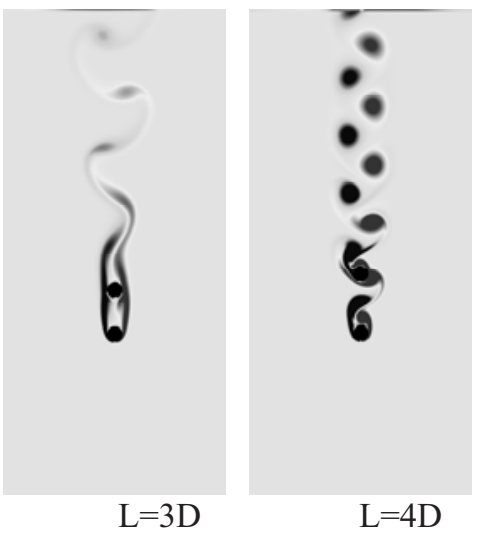

Fig. 3. Vorticity patterns showing the wake interference for several values of $\mathrm{L}$. $\mathrm{Re}=200$ 


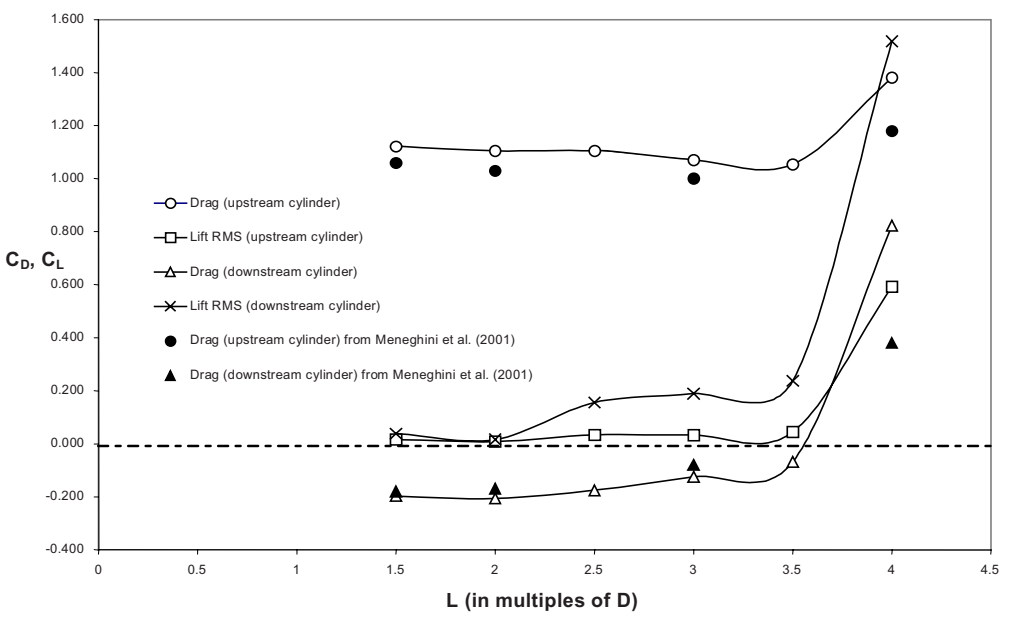

Fig. 4. Drag and lift coefficients on the two cylinders. For L smaller than 3.5D, the drag force is negative on the downstream cylinder. $\mathrm{Re}=200$

Fig. 5. gives the Fourier transform $\mathfrak{I}\left[F_{y}(T)\right]=\hat{F}_{y}(f)$ of the lift force against the Strouhal number, $S t=f D / U$, for $\mathrm{L}=2 \mathrm{D}$. The vortices are produced with a single dominant frequency in all the simulations. In this case a second dominant frequency appears, showing a growing frequency at $\mathrm{St}=0,13$ (which is the unique dominant frequency for $\mathrm{L}=3 \mathrm{D}$ ).

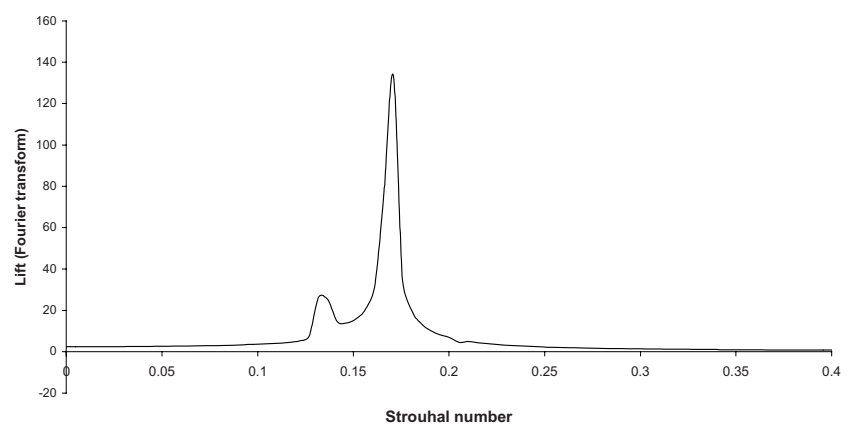

Fig. 5. Fourier transform of the lift force against the Strouhal number for $L=2 D$

\subsection{Two Identical Circular Cylinders Aligned Orthogonal to the Main Flow Direction}

Fig. 6 shows the simulation domain and the geometric parameters that were used in the LB simulation. Distance L between the centers of the two cylinders, referred as 1 and 2 was varied from $1.5 \mathrm{D}$ to $4 \mathrm{D}$. 


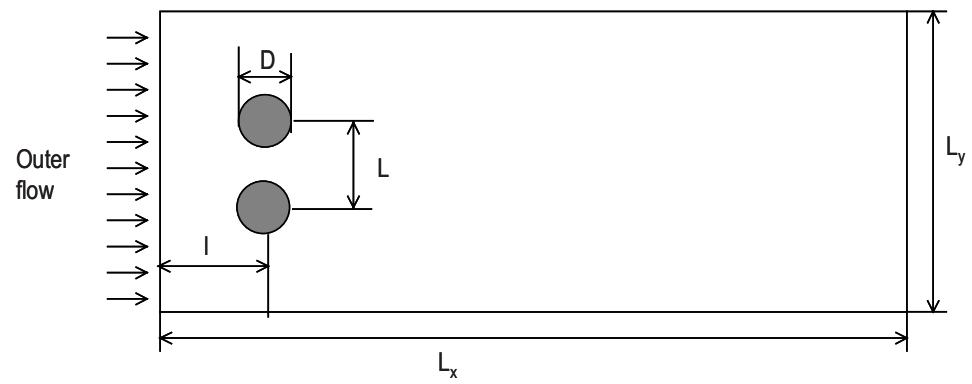

Fig. 6. Simulation domain for the fluid flow against two cylinders aligned orthogonal to the main flow. $\mathrm{L}_{\mathrm{x}}=30 \mathrm{D}$ (1200 lattice sites), $\mathrm{L}_{\mathrm{y}}=21 \mathrm{D}$ (820 lattice sites), $\mathrm{D}=40$ lattice sites. Distance $\mathrm{L}$ between the centers of the two cylinders was varied from $1.5 \mathrm{D}$ to $4 \mathrm{D}$

Fig. 7 shows the vorticity patterns for several $\mathrm{L}$. When $\mathrm{L}$ is smaller than $\sim 3 \mathrm{D}$, the wake interference becomes increasingly important and the two cylinders have opposed lifts that try to separate one from another. This can be seen in Fig. 8, which presents the simulation results for the drag, $\bar{C}_{D}$ and lift, $\bar{C}_{L}$, coefficients, for the two cylinders. Drag and lift results were compared with Meneghini et al., [5]. Agreement between the two methods can be considered as very good, for the lift coefficient, although present LB simulation has overestimated the drag coefficient with respect to Meneghini and co-authors' results. For this Lattice Boltzmann simulation, a single $1200 \times 820$ lattice size was used and Lattice Boltzmann results have to be considered as preliminary.

Figs. 4 and 8 show some overestimation of LB simulation for the drag force when compared to Meneghini et al.'s results, [5], indicating that more extensive LB simulation is still to be carried out, for clarifying this disagreement. Boundary conditions can be, further, improved beyond the presently used bounce-back condition to reduce discretization effects in the geometrical representation of the circular cylinder surface.

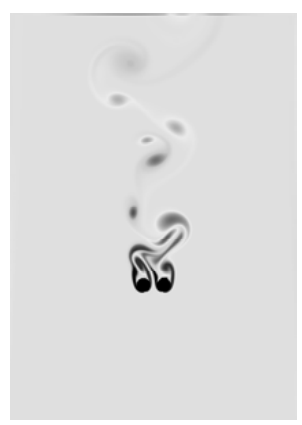

$\mathrm{L}=1.5 \mathrm{D}$

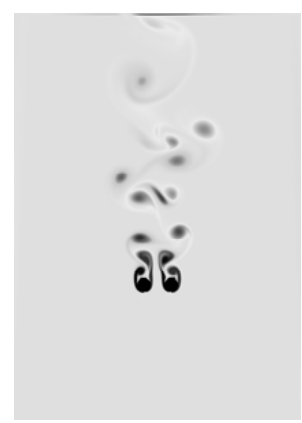

$\mathrm{L}=2 \mathrm{D}$

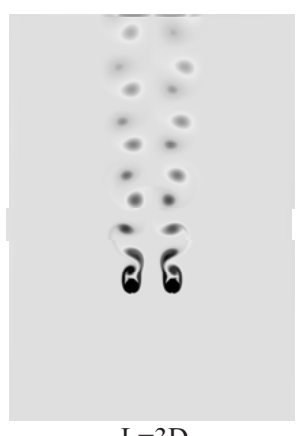

$\mathrm{L}=3 \mathrm{D}$

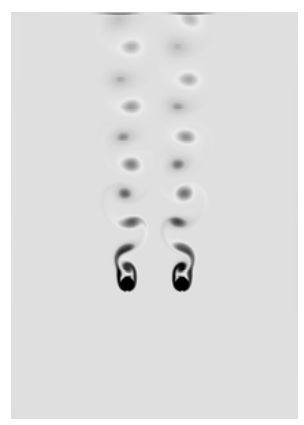

$\mathrm{L}=4 \mathrm{D}$

Fig. 7. Vorticity patterns showing the shedding interference for several values of $\mathrm{L}$. $\mathrm{Re}=200$ 


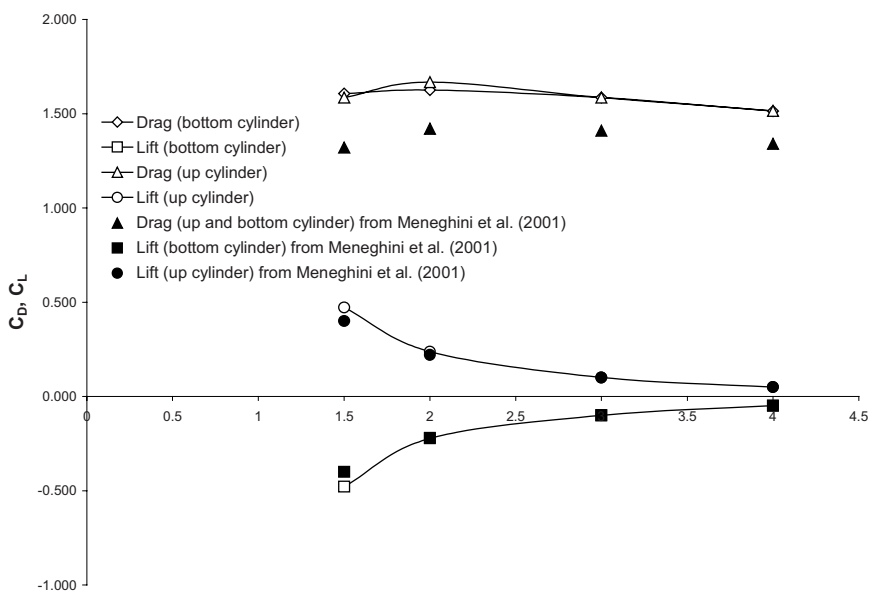

L (in Multiples of D)

Fig. 8. Drag and lift coefficients on the two cylinders. Lift coeficients are opposed and try to separate the two cylinders. $\mathrm{Re}=200$
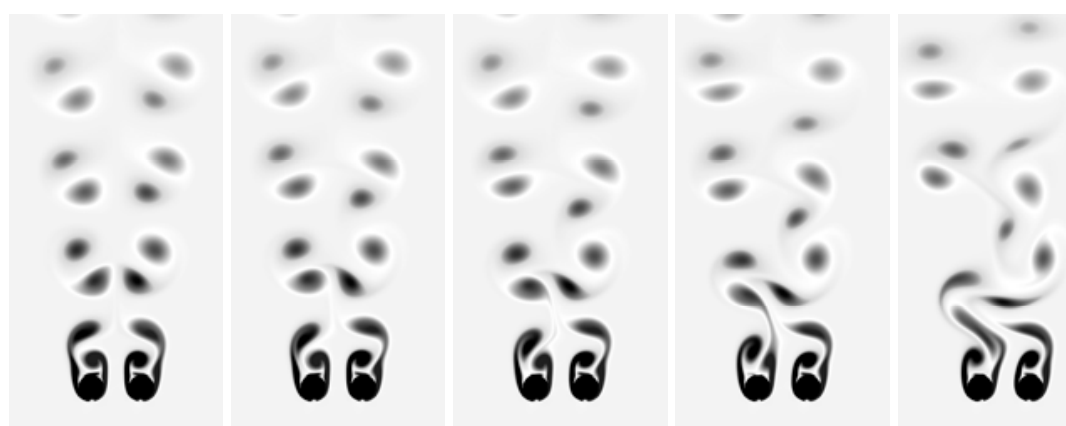

Fig. 9. Time sequence of vorticity images showing the beginning of the wake interference for $\mathrm{L}=1.5 \mathrm{D}$. Images were obtained at time-step intervals corresponding to a single period of vortex formation. $\mathrm{Re}=200$

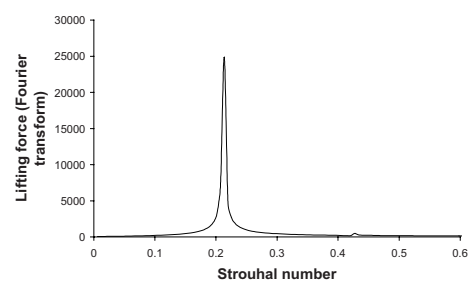

$\mathrm{L}=3 \mathrm{D}$

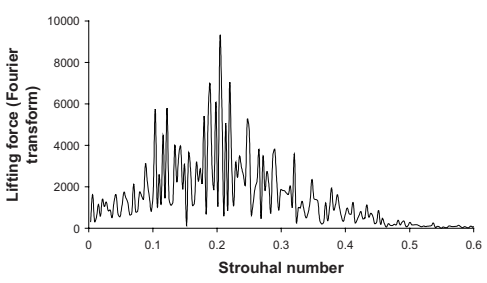

$\mathrm{L}=1.5 \mathrm{D}$

Fig. 10. Strouhal number, $S t$, spectrum for $L=3 D$ and for $L=1.5 D$. When $L$ is smaller than $\sim 2 D$ vortices are formed and released with a continuous frequency spectrum, indicating a flow transition to turbulence. When $\mathrm{L}$ increases above $\mathrm{L} \sim 2 \mathrm{D}$, vortices are formed with a single frequency related to $\mathrm{St}=0.21$ 
Fig. 9 shows a time-sequence of vorticity images showing when wake interference begins and flow pattern changes to a transition pattern. This is clearly seen in Fig. 10, where the Strouhal spectrum for $\mathrm{L}=3 \mathrm{D}$ and for $\mathrm{L}=1.5 \mathrm{D}$, are compared. When the two cylinders are located at a distance $\mathrm{L}$ larger than $2 \mathrm{D}$, vortices are formed with a single dominant frequency. When these bodies are neared, flow interference enlarges the spectrum frequency with which the vortices are formed and released to the main flow, driving a transition regime to turbulence. The frequency spectrum that was obtained for the case $\mathrm{L}=2 \mathrm{D}$ was similar to the one for $\mathrm{L}=1.5 \mathrm{D}$, but less broad and with a more clear predominance of $\mathrm{St}=0.21$.

\section{Conclusions}

In present paper, a lattice-Boltzmann method was used for studying the flow interference, when two solid bodies are immersed in the flow, at near locations. Simulation results were compared with the results obtained with the Fraction Step Method.

The results for a single circular cylinder placed in the flow sugests that this method can be applied to simulate complex flows in the range of Re from 50 to 200 .

Main conclusion are: i) when the cylinders are aligned along the main-flow direction, vortices are formed with a single frequency for $\mathrm{Re} \leq 200$; ii) transition to turbulence appear in two-dimensional flow, when the cylinders are aligned orthogonal to the main flow direction and separated by distances smaller than $\sim 2 \mathrm{D}$.

It is well known, [2,3], that for a single circular cylinder, three-dimensional vortices appear above $\mathrm{Re}=200$. This is the main reason, present work was limited to $\mathrm{Re}=200$. Nevertheless, flow interference produced by two or more solid bodies can give rise to the development of three-dimensional vortices at lower Reynolds number. In this way, further work must consider three-dimensional LB simulations.

\section{Acknowledgements}

Authors are greatly indebted with CNPq (Brazilian Council of Scientifical and Technological Development), ANP (Brazilian National Agency of Petroleum) and Finep (Fundação Nacional de Estudos e Pesquisas) for the financial support.

\section{References}

1. Lange, C. F., Durst, F., Breuer, M.: Momentum and Heat Transfer from Cylinders in Laminar Crossflow at $10^{-4}<=\operatorname{Re}<=200$. Int. J. of Heat and Mass Trans. 41 (1998) 34093430

2. Thompson, M.C., Leweke, T., Williamson, C.H.K.: The Physical Mechanism of Transition in Bluff Body Wakes. Journal of Fluids and Structures 15 (2001) 607-616 
3. Williamson, C.H.K.: Advance in Our Understanding of Vortex Dynamics in Bluff Body Wakes. Journal of Wind Engineering and Industrial Aerodynamics 69-71 (1997) 3-32

4. Lima e Silva, A.L.F, Surmas, R., dos Santos, L.O.E., Philippi, P.C., Silveira Neto, A.: Comparative Numerical Simulation of Two-Dimensional Flows Over a Pair of Circular Cylinders, Disposed in Tandem, Using Immersed Boundary and Lattice-Boltzmann Methods. In: Conference On Bluff Body Wakes And Vortex Induced Vibrations BBVIV3, 17-20 December 2002, Port Douglas, Australia

5. Meneghini, J.R., Saltara, F., Siqueira, C.L.R., Ferrari Jr, J. A.: Numerical Simulation of Flow Interference Between Two Circular Cylinders in Tandem and Side-by-Side Arrangements. Journal of Fluids and Structures 15 (2001) 327-350

6. Williamson, C.H.K.: Evolution of a Single Wake Behind a Pair of Bluff Bodies. Journal of Fluid Mechanics 159 (1985) 1-18

7. Bhatnagar, P.L., Gross, E.P., Krook, M.: A Model for Collision Processes in Gases. I. Small Amplitude Processes in Charged and Neutral One-Component System. Physical Review 94 (1954) 511-525

8. He, X., Luo, L.S.: Theory of Lattice Boltzmann Method: from the Boltzmann Equation to the Lattice Boltzmann Equation. Physical Review E 56 (1997) 6811-6817

9. Mcnamara, G.R., Zanetti G.: Use of the Boltzmann Equation to Simulate Lattice-Gas Automata. Physical Review Letters 61 (1988) 2332-2335

10.Qian, Y. H., d'Humières D., Lallemand P.: Lattice BGK Models tor Navier-Stokes Equation. Europhys. Lett. 17 (1992) 479-484

11.He, X., Luo, L.S., Dembo, M.: Some Progress in Lattice Boltzmann Method: Part I. Nonuniform Mesh Grids. Journal Of Computational Physics 129 (1996) 357-363

12.Bouzidi, M., D’Humières, D., Lallemand, P., Luo, L.S.: Lattice Boltzmann Equation on a Two-Dimension Rectangular Grid. Journal of Computational Physics 172 (2001) 704-717

13.Park, J., Kwon, K., Choi, H.: Numerical Solutions of Flow Past a Circular Cylinder at Reynolds Number Up to 160. KSME International Journal 12 (1998) 1200

14.Sucker, D., Brauer, H.: Fluiddynamik bei der angestromten Zilindern. Wärme und Stoffubertragung 8 (1975) 149

15.Lima e Silva, A. L. F., Silveira-Neto, A., Damasceno, J. J. R.: Numerical Simulation of Two Dimensional Flows over a Circular Cilynder using the Immersed Boundary Method. submitted to Journal of Computational Physics (2002)

16.Williamson, C. H. K.: Oblique and parallel modes of vortex shedding in the wake of a circular cylinder at low Reynolds numbers. J of Fluid Mech 206 (1989) 579-627

17.Barkley, D., Henderson, R. D.: Three-dimensional Floquet stability analysis of the wake of a circular cylinder. Journal of Fluid Mechanics 332 (1997) 214-241

18.Karniadakis, G. E., Triantafyllou, G. S.: Frequency selection and asymptotic states in laminar wakes. Journal of Fluid Mechanics 199 (1989) 441-469 